\title{
1 Knowledge organisation for digital humanities
}

\author{
An introduction
}

\author{
Koraljka Golub, Ahmad M. Kamal, \\ and Johan Vekselius
}

\section{Introduction}

The field of digital humanities (DH), which emerged as the umbrella term in the mid-2000s for humanities scholarship using computational techniques, has hosted many contentious discussions on the field's identity and purview, with think pieces positing "what DH means" and "what counts as DH" becoming a genre of its own (Kirschenbaum 2010). As the field has continued to develop since then, settling on a familiar assemblage of techniques, concepts, schools, tools and so on, it has nevertheless remained open to new opportunities for further exploration and growth. One avenue we see for the advancement of DH is a deeper engagement with the discipline of knowledge organisation (KO). Some aspects of $\mathrm{KO}$ have appeared piecemeal within the greater library of DH endeavours; as a result, introductory DH materials commonly include some facets of KO, e.g., the chapter on metadata in Drucker's coursebook (2021). Nevertheless, KO is not commonly engaged with head-on within the field. The wider repertoire of understandings, skills and resources that $\mathrm{KO}$ has to offer has been overlooked by the wider community of DH scholars. This volume addresses that gap by providing a range of international perspectives and approaches which reiterate the value of $\mathrm{KO}$ for the $\mathrm{DH}$.

$\mathrm{KO}$ is a major discipline within the field of information science, and is largely concerned with the practices of institutions such as libraries, archives and museums (LAMs) as they organise, catalogue and classify resources for communities of users (e.g., researchers, students, the public or company staff). Yet the need to systematically organise information has become universal in the digital era. Ad hoc solutions from those unfamiliar with KO to manage new collections of data can suffer from problems of sustainability, scalability or tractability: these are challenges that DH researchers commonly face, as variously documented in some of the case studies presented in this volume. We argue that bringing together the understanding of the $\mathrm{KO}$ community and ambitions of the $\mathrm{DH}$ community can help provide strategic solutions to the challenge of managing information, thereby supporting better information retrieval systems, improving the stewardship of 
data, creating more venues for information access, and discovering more meaningful information.

To support our argument for the importance of $\mathrm{KO}$ in $\mathrm{DH}$, this book presents examples of interdisciplinary and cutting-edge DH case studies in which the practice of organising information is put at the forefront. The contributions by 41 authors, from 16 countries across 4 continents, represent an international range of perspectives, challenges and solutions. By exploring the various findings, the book posits a future research agenda for the field of $\mathrm{DH}$ where the role of $\mathrm{KO}$ is more clearly delineated, both in identifying the long-standing issues in $\mathrm{DH}$ which $\mathrm{KO}$ can help address and how DH scholars and practitioners could incorporate $\mathrm{KO}$ moving forward. This, we hope, will set the stage for a new transdisciplinarity in $\mathrm{DH}$, a field whose defining characteristic since its inception - beyond its adoption of digital methods - has been in a constant search for partnerships and collaborations to realise new opportunities for knowledge production and sharing.

This chapter aims to highlight on a general level the relevance of $\mathrm{KO}$ for the $\mathrm{DH}$, thereby providing an overarching context for the book and integrating the various contributions within it. After briefly introducing the fields of $\mathrm{KO}$ and $\mathrm{DH}$, the chapter reviews how the two fields intersect, both in regards to work in $\mathrm{DH}$ in the cultural heritage sector and in academic scholarship (Background). It specifically focusses on those themes which are reflected in the contributed book chapters, summaries of which are then presented (Chapters overview). The end of the chapter (Moving forward) distils two constantly recurring issues in $\mathrm{DH}$, based on the contributions, information discovery and information representation, which we argue $\mathrm{KO}$ is well positioned to help us address.

\section{Background}

\section{Knowledge organisation and related research fields}

The discipline of $\mathrm{KO}$ "is about describing, representing, filing and organising documents and document representations as well as subjects and concepts both by humans and by computer programs" (Hjørland 2016a). The practices of KO are omnipresent in people's lives, from the shopping list to the legal code and implements different types of KO systems (lists, subject headings, thesauri, taxonomies, classifications, catalogues etc.).

In order to support the organisation of information resources, the field of $\mathrm{KO}$ creates various standards and guidelines to create representations of information objects (e.g., library books, archival documents museum artefacts) such as those held by LAMs. The resulting representations are called metadata or data about data. For more nuanced definitions and discussions thereof, see Mayernik (2020); for an in-depth overview of related standards, see Zeng and Qin (2016). Examples of standards for data elements include cataloguing guidelines such as Resource Description and Access (RDA) 
used by libraries, Cataloging Cultural Objects (CCO) used by museums and Describing Archives: A Content Standard (DACS) for archives. Standards for data values encompass controlled vocabularies such as the Library of Congress Subject Headings (LCSH) or the Dewey Decimal Classification (DDC), which offer consistency in assigning subject headings and classifying materials, respectively. While many of the standards were first created before the advent of computers, they have been adapted to meet the new demands for enabling data exchange. In fact, many of the developments in cultural heritage metadata in recent decades have been driven by the transition from paper catalogue records to online catalogues, especially with the establishment of the World Wide Web.

Another term for $\mathrm{KO}$ is information organisation (cf. Hjørland 2012; Hjørland 2016a), and in this volume the two terms are used interchangeably. The distinction between knowledge and information themselves, along with the related terms data and wisdom, is frequently discussed in the literature (for an overview, see Bates 2010). The ever-popular Data-InformationKnowledge-Wisdom (DIKW) pyramid is often used to illustrate a hierarchical relationship between the four concepts, from the least processed (data) to the most processed and contextual (wisdom). But the distinctions between these concepts, as well as the assumptions behind them, carry significant implications which have been the subject of contention. For instance, the perception of data as a "raw" descriptive unit has been well deconstructed (see Gitelman 2013), with prominent voices within DH suggesting the notion of capta in the place of data to emphasise the active interpretation that goes into the constitution of data (Drucker 2011). In turn, Oldman (Chapter 7) criticises any information system based on "data" as being ineffective for $\mathrm{DH}$, especially for historical research and, as such, he calls for $\mathrm{KO}$ approaches based on textual narratives to facilitate knowledge creation and mediation.

$\mathrm{KO}$ is an important subfield of information studies or information science which is "the science and practice dealing with the effective collection, storage, retrieval, and use of information" (Saracevic 2009). Closely related to $\mathrm{KO}$ are several other research fields and disciplines. One is information retrieval (IR), which is focussed on developing and evaluating computational systems that provide information to address a user's search requirements (see Glushko 2016); like KO, IR is a separate sub-discipline within information science, but much of the research in IR occurs within the field of computer science as well, focussing on designing and modelling retrieval systems. Another related area within information science is information behaviour (IB), which investigates people's information needs, seeking, using and sharing it across different contexts and roles (Case and Given 2016). Human computer interaction (HCI), which studies the design of computer interfaces for optimal user experience, intersects with several disciplines, including information science. $\mathrm{HCI}$ is relevant to $\mathrm{KO}$ given that user interfaces play an important role in how effectively $\mathrm{KO}$ and IR systems 
can be leveraged. We regard all these fields as being closely related because they all contribute to understanding the complexities surrounding $\mathrm{KO}$, IR and information use. In order to improve information access in general and in interdisciplinary fields such as the $\mathrm{DH}$ in particular, it is important to employ KO correctly in conjunction with each related area of research.

\section{Digital humanities}

The Oxford English Dictionary defines DH as "an academic field concerned with the application of computational tools and methods to traditional humanities disciplines such as literature, history, and philosophy" ("Digital humanities", 2020). While adequate, this definition belies the continuous discussion and (re-)formulation by scholars and practitioners across a range of disciplines in how to describe the field (see Terras, Nyhan, and Vanhoutte 2013). Regardless, the field of DH is understood to sit at the intersection of computation and the humanities. Its roots are in the field of humanities computing, with early work reaching as far back as the 1940s (Busa 1980). The name "digital humanities" itself emerged in the early 2000s as a more representative and contemporary designation for the field (Vanhoutte 2013; Nyhan and Flinn 2016).

In this book $\mathrm{DH}$ is discussed in its broad sense while taking into consideration specific affordances, as set out in the definition formulated by Gardiner and Musto $(2015,4)$ : "harnessing computing power to facilitate, improve, expand and perhaps even change the way humanists work". Since, as noted above, the field is at the crossroads of the humanities and computation, DH unites traditional humanities disciplines such as archaeology, history, philosophy, linguistics, literature, art, music and cultural studies with computing tools and techniques, e.g., hypertext/media, data visualisation, IR, statistics, 3D modelling, data/text mining, digital mapping and spatial analysis. But beyond DH scholars using computational methods to answer traditional research questions, we also see them developing new perspectives and new questions that can be solved through pioneering methods brought about by the digital transformation of knowledge creation (Kansa and Kansa, 2021). For an overview of DH and its concepts, technologies and methods, see Schreibman, Siemens, and Unsworth (2016) or Drucker (2021).

A consequence of DH's hybridity is the new-found importance of infrastructures and collaborations. Bringing together traditional scholarship and cutting-edge technical expertise, while also leveraging new repositories of digital assets, entails greater coordination and cooperation between individuals and institutions than is typical in humanities scholarship. It is therefore unsurprising to see LAMs playing a prominent role both in supporting DH scholarship and also undertaking their own DH initiatives. And it is this same impetus for the provision of digital cultural heritage (whether for scholarship or outreach) where the relevance of $\mathrm{KO}$ for $\mathrm{DH}$ can be most clearly seen. 


\section{Knowledge organisation for digital humanities}

In the cultural heritage sector, $\mathrm{KO}$ has always been an essential component of managing collections. But $\mathrm{KO}$ also takes on a new level of relevance in the field of $\mathrm{DH}$, both for those within LAMs who are already intimately familiar with $\mathrm{KO}$ systems and for scholars whose interactions with $\mathrm{KO}$ are generally quite limited, e.g., to conducting literature searches in library databases. The difference between those within $\mathrm{DH}$ working in the cultural heritage sector and those working in pure scholarship is compounded by the different orientations of their respective $\mathrm{DH}$ projects: in the cultural heritage sector DH initiatives are usually aimed at expanding access to cultural objects, whereas specific research questions shape DH projects in academia. Given these distinctions, we discuss each context in turn: first $\mathrm{KO}$ for $\mathrm{DH}$ within the cultural heritage sector, and then its role within academic research.

\section{Within cultural heritage}

We have already mentioned some examples of KO systems that have been developed for LAMs over the years. Today, the cultural heritage sector is once again responding to a dramatic shift in its information infrastructure in the form of the Semantic Web (Web 3.0), as an extension of the World Wide Web (Berners-Lee, Hendler, and Lassila 2001). The Semantic Web is envisioned to go beyond simply linking web pages for human navigation to linking entire datasets, thereby allowing computers to connect and disambiguate data or generate novel information. One of the foundational technologies in this ambitious endeavour is internationalised resource identifiers (IRIs) that uniquely identify concepts for people, objects, epochs, genres etc., represented by different terms. For instance, Mahatma Gandhi (also Mohandas Karamchand Gandhi, મોહનદાસ ગાંધી etc.) could be represented as a machine-readable IRI (https:/viaf.org/viaf/71391324/); the same could be done for New Delhi (http://vocab.getty.edu/tgn/7001534) and the practice of nonviolent resistance (https://dbpedia.org/resource/ Nonviolent_resistance).

Semantic Web technologies like IRIs and related standards allow for the aggregation of metadata from LAMs in order to access cultural heritage across otherwise isolated collections. However, data aggregation preceded the advent of IRIs. One example is Social Networks and Archival Context (SNAC), an international cooperative of archives, libraries and museums combining archival records with authority files from different institutions. Today the largest data aggregator for LAMs in Europe, Europeana, provides access to over 50 million digitised objects (Europeana 2021). Beyond facilitating data aggregation initiatives, Semantic Web technologies also allow datasets of cultural heritage objects to be linked to external data from across the Web, a concept known as linked data (LD), as defined by 
the Word Wide Web Consortium (2015). Furthermore, if such data is made openly available, linked open data (LOD) is created.

Semantic Web technologies and LOD also allow metadata to be enriched by other metadata, a process known as semantic metadata enrichment. Europeana enriches its data providers' metadata by automatically linking text strings found in the metadata to controlled terms from linked open datasets or vocabularies (W3C Incubator Group 2011). In this way, further links across the Web are established and resources from datasets in different databases become linked. Semantic Web technologies also help make data from various cultural heritage institutions FAIR: findeable, accessible, interoperable and reusable (Wilkinson et al. 2016). LAMs strive to make their metadata available as LOD, FAIR, semantically enriched and aggregated with other LAMs' data in order to help make cultural heritage easily discoverable and openly available to all.

Interestingly, from the perspective of $\mathrm{KO}$ in the cultural heritage sector, the Semantic Web was in fact keeping in line with the ongoing developments. The 1990s witnessed efforts from LAM communities to create universal conceptual models for information object representations, spurred on by the transfer to a digital and networked world, rather than by representations based on any specific type of cultural heritage institution or any specific collection. These conceptual models represent metadata at the highest level of abstraction, articulated through entities (e.g., the person entity Mahatma Gandhi; the work entity "The Story of My Experiments with Truth") and relationships (e.g., Mahatma Gandhi is the creator of "The Story of My Experiments with Truth"). This simple entity-relationship model, which also incorporates attributes or properties, allows for very sophisticated KO networks whereby users can more readily identify, find, select and obtain cultural resources. In the libraries community, this is embodied by the Functional Requirements for Bibliographic Records (FRBR) family of conceptual models for catalogue functionality, which were consolidated into the IFLA Library Reference Model (IFLA LRM, International Federation of Library Associations 2017). In the museums sector, the standard corresponding to the IFLA's LRM is the ISO standard CIDOC-CRM (CIDOC Conceptual Reference Model; International Standards Organization 2006) developed by the International Committee for Documentation of the International Council of Museums (ICOM). Similarly, in archives, the conceptual model is Records in Context: A Conceptual Model for Archival Description (RiC, International Council on Archives 2019).

\section{Within academic research}

Apart from its application in the cultural heritage sector, KO offers other means for supporting DH research. This includes enriching digital objects (textual or non-textual), automated analyses of content, the documentation 
and organisation of DH projects and their various outputs, or even the representation of humanistic knowledge itself.

As said at the outset, $\mathrm{KO}$ is in essence concerned with systematic representations of information resources. Traditionally, this has meant itemlevel or document-level descriptions (books, music scores, tapestries etc.). However, the shift to digital resources has allowed for more granular levels of representation to be embedded within the documents themselves. Standard generalised markup language (SGML) was developed in the 1980s as a means of structuring digital publications, with predefined tags included within the content of a document. These tags, instead of conveying explicit content, provided structural and formatting information. SGML was also adapted as a core technology for the Web in the hypertext markup language (HTML) which, when embedded in the content of a webpage, conveys instructions on how the page was to be displayed in a browser. Another derivation of SGML, extensible markup language (XML), is even simpler and carries no predefined tags. As such, it offers a highly flexible system for further enriching and describing the contents of documents according to any schema one might wish to develop. In cultural heritage institutions XML is a commonly used standard; an example is Encoded Archival Description (EAD) used for encoding archival finding aids.

DH scholars were quick to adapt these tools. For instance, textual scholarship in DH took up XML to markup (or encode) texts, and also collaborated in the development of an XML standard called the Text Encoding Initiative (TEI, Text Encoding Initiative 2017). TEI offers systematic guidelines for representing various aspects inherent within the texts that would be of interest to linguists, literary scholars, historians, philologists, dramatists and so on, whether that be a rhyme scheme of a poem, variations in spelling, references to a specific person or place etc.. As such, text encoding methods became a common standard in the humanities to explicitly structure the contents of texts by marking up constituent parts of a text. So rather than just document-level metadata typical of LAMs, metadata could be applied at the level of a chapter, page, paragraph, sentence, line, word or letter. But while TEI involves a novel degree of KO than was hitherto common in cultural heritage institutions, it has been adopted by some LAMs. Flanders and Jannidis (2018) see a clear distinction between applications of TEI (and other data models) for curation-driven activities, stressing access and preservation (see TEI for Libraries, Hawkins et al. 2018) and the application of TEI for research-driven activities (where research projects determine the assets of interest). For an overview of markup, see Chapter 4 of Druckner (2021).

Another area where KO dovetails with DH scholarship has developed from forays into automated information organisation. Efforts in libraries to automate the analysis and organisation of resources share a similar history with the efforts to apply computational text analysis for DH research. They are connected to the use of punch cards; e.g., Kilgour (1939) describes their use for library circulation records. Roberto Busa's plans to encode 
nearly 11 million words of Thomas Aquinas' writings on IBM punch cards back in 1946 is often considered as the origin of DH (Sula and Hill 2019). In 1959, Busa's resulting concordance, an alphabetical list of words with their immediate context, inspired an information scientist at IBM, H. P. Luhn, to design the Key-Word-in-Context (KWIC) index, in which each word is presented together with its surrounding words (Haeselin 2017; Luhn 1960). In contrast with the traditional system of bibliographic indexes, KWIC offered a new, automated means of creating indexes based on the extracted contents of technical documents: in other words, a revolutionary new KO system In the decades that followed, $\mathrm{KO}$ has continued exploring ways of leveraging computation to move beyond manual information organisation towards semi-automated or fully automated approaches. A common application area is automatic subject indexing (for an overview, see Golub 2019) or metadata generation (Golub, Muller, and Tonkin 2014).

These automated approaches to representing documents also connect with a domain of DH that focusses on textual scholarship (Sula and Hill 2019). Applying computational methods to study massive corpora of literary texts has been referred to by Moretti (2000) as distant reading, which he contrasts with the slow and partial insights gleaned from the close reading typical of traditional literary scholarship. For instance, one could mine literary texts for emotional words ("sad", "forlorn", "rage", "joyous" etc.) to trace the predominance of particular classes of sentiments over historical periods or around historical events: see Acerbi et al. (2013) for an example; see Chapter 7 of Drucker (2021) for an overview. A common computational method is topic modelling to automatically extract topics in a collection of documents, which is especially useful for "reading" a large number of humanities texts and discovering hidden themes: see Blei (2012) for an example.

Finally, if we shift our attention from the digital methods applied within a DH project to long-term sustainability and access to the project itself, it is once again necessary to recognise $\mathrm{KO}$ as crucial for supporting the availability of digital research outputs like research data throughout their life cycle. Other digital research outputs that are particularly complex and challenging to maintain are websites, databases and interactive visualisation tools. Ensuring continued access and re-use of such outputs require metadata, KO procedures and technical solutions, not to mention funding; these challenges are addressed by Kräutli, Chen and Valleriani (Chapter 10).

\section{Chapters overview}

This book reflects the dominant research activities at the intersection of $\mathrm{KO}$ and $\mathrm{DH}$. The volume is structured into the following main themes: Modelling and Metadata (Part I), Information Management (Part II) and Platforms and Techniques (Part III). 


\section{Part I: Modelling and metadata}

The first part of the book, Modelling and Metadata, consists of six chapters which address the challenges of modelling cultural heritage data, harmonisation of conceptual models, approaches to metadata aggregation and metadata enrichment and the need to move from organising data to organising knowledge.

Engaging with the diversity of collections of digital objects in various cultural domains, Chapter 2, "Modelling cultural entities in diverse domains for digital archives", by Shigeo Sugimoto, Chiranthi Wijesundara, Tetsuya Mihara and Kazufumi Fukuda, is concerned with the construction of generalised data models for cultural heritage data, including intangible cultural heritage and new media arts. This work discusses digital archiving processes from the perspectives of model entity types (such as conceptual and embodied entities) and proposes metadata models for new media artworks and performing arts. It highlights the importance of well-organised data models for interoperability across domains, a recurring theme in several chapters (see Chapters 3 and 4). These data models are generalised to serve as a framework that is neutral towards application domains and can be used in combination with domain-oriented models such as CIDOC CRM for museums and IFLA LRM for libraries, echoing Vukadin and Štefanac in the following chapter. The authors emphasise that accurate modelling and clarification of types of entities is essential for the accurate identification of entities in the implementation of LD.

Chapter 3, "Collection-level and item-level description in the digital environment: Alignment of conceptual models IFLA LRM and RiC-CM", by Ana Vukadin and Tamara Štefanac, is an attempt to harmonise two conceptual data models for digitised historical resources. This work addresses the question of a cross-domain scheme at the intersection of digital scholarship and library and archival practices. Specifically, the case study demonstrates the harmonisation of the IFLA LRM and RiC, which brings together two different levels of data models from each respective field: the collection-level description of archiving with the item-level description of librarianship, thereby encompassing a wider range of descriptive granularity and abstraction/materiality. The resulting model allows for flexibility and implementation in various environments. It is crucial that metadata schemes are "scalable" according to the principle of functional granularity to support different needs and for metadata requirements to be met at different levels of description. The authors call for simple and straightforward guidelines based on models and standards from established communities in order to ensure uptake by DH projects with little expertise in information organisation.

Metadata aggregation is an important approach to facilitating resource discovery in cultural heritage. Chapter 4, "Linked Open Data and aggregation infrastructure in the cultural heritage sector: A case study of $\mathrm{SOCH}$, 
a Linked Data aggregator for Swedish open cultural heritage", by Marcus Smith, introduces LOD and metadata aggregation on both a theoretical and a technical level, introducing key concepts and standards, as well as giving a practical example in the form of the SOCH (Swedish Open Cultural Heritage) LD aggregator platform. The SOCH service implements the FAIR principles and LOD technologies to serve as the Swedish data infrastructure for the museums, archives and historic environment registers to share metadata. The metadata are mapped to a common data model and are made queryable via an application programming interface (API). Over its ten-year lifespan, SOCH has had significant success in opening up Swedish heritage data. However, as an early adopter of LOD in Swedish heritage since its establishment in 2008, SOCH has faced some challenges because it failed to keep up with developing standards within LOD. Its use of a bespoke data model and API now presents an obstacle to interoperability and integration with the wider Semantic Web.

Another approach to bolstering resource discovery of cultural heritage data is semantic enrichment. Chapter 5, "A Semantic enrichment approach to linking and enhancing Dunhuang cultural heritage data", by Xiaoguang Wang, Xu Tan, Heng Gui and Ningyuan Song, features a work on semantic enrichment which focusses on the construction of the Dunhuang Mural Thesaurus (DMT) by using natural language processing (NLP) techniques along with the domain knowledge of experts in the field. Incorporating semantic analysis, linking and augmentation, the thesaurus was ultimately published as LOD in order to support cultural studies of Dunhuang. It proposes future research in user studies to further improve its KO platform.

While large national institutions have been at the forefront of digitisation, smaller organisations have lagged behind. Chapter 6, "Semantic metadata enrichment and data augmentation of small museum collections following the FAIR principles", by Andreas Vlachidis, Antonis Bikakis, Melissa Terras and Angeliki Antoniou, is thus an important case study of how a small museum created a digital collection to improve access to the museum's artefacts.. This work emphasises the FAIR principles for sharing data widely and the application of semantic models and methods such as the selection of an underlying ontology and semantic enrichment to include historic reflections and interpretations of the data. Highlighting the importance of interoperability, the semantically enriched collection of data links to entities from external data sets. For smaller museums where digitisation of complete collections is impractical, semantically enriching the collection at hand with LOD offers a feasible alternative, by leveraging pre-existing digital assets within external repositories. A challenge that such a project faces, however, is the need to coordinate between the necessary actors (museum staff, DH researchers and computer scientists) who each bring a different discipline-specific understanding of data and information.

Earlier in this chapter we introduced the DIKW pyramid and the manner in which it makes data the basis of all other epistemic levels. In Chapter 7, 
"Digital research in the humanities and the legacy of form and structure", Dominic Oldman makes an important and constructive critique of this data-driven approach to the organisation of information within DH. The chapter argues that the data-driven epistemology is fostered by the logic of commercial databases that have been imposed upon the humanities. Thus, DH tends to model and organise data in ways that poorly correspond to the needs of researchers themselves, failing to deliver on the promise of complementing humanistic inquiry with appropriate computational techniques. In a counterexample to this trend, Oldman presents the ResearchSpace system developed at the British Museum. As a departure from the typical design principles of such a system, ResearchSpace is concerned with contextualised representations of data using the textual narrative as a metaphor for how to structure and organise data. The idea is to create a platform that better captures and expresses different ways of thinking, various historical contexts and a diversity of knowledge in a manner that corresponds to researchers' requirements. ResearchSpace demonstrates the need for meaningful $\mathrm{KO}$ in $\mathrm{DH}$, where relevant models for the representation of information and knowledge must be advanced, especially in the face of information models based on a narrow, technological world view instantiated in database management systems and LD.

\section{Part II: Information management}

The second part of the book, Information Management, is made up of three chapters which discuss the handling of different assets in DH research: the texts being studied, the research tools being developed and the outputs which are published (websites, scholarly editions etc.).

Chapter 8, "Research access to in-copyright texts in the humanities", by Peter Organisciak and J. Stephen Downie, is concerned with the management of resources for quantitative text analysis, specifically with regard to copyright concerns. The chapter proposes the principle of non-consumptive access. The case study explores how the HathiTrust Research Center constructed a dataset based on a massive digital library that allows researchers to do advanced quantitative text analyses of corpora without accessing the copyrighted text, to enable distant reading. The chapter thus demonstrates a practical solution to the highly relevant problem of copyright in the context of DH and KO.

Chapter 9, "SKOS as a key element for linking lexicography to digital humanities", by Rute Costa, Ana Salgado and Bruno Almeida, explores the relationship between $\mathrm{DH}$, information science and lexicography through the lens of the digitisation of a Portuguese legacy dictionary. This chapter discusses the construction of lexical resources encoded according to TEI and using an information structure based on the Simple Knowledge Organisation System (SKOS), which enables the dictionary to be connected to other vocabularies. The project has proved fruitful, as its methodology 


\section{Koraljka Golub et al.}

will inform the digitisation of other legacy dictionaries. One key challenge raised by the chapter is to combine the skills of the various scientific disciplines that together make up the humanities with those of information science in order to deliver a high standard of service for stakeholders.

Chapter 10, "Linked Data strategies for conserving digital research outputs: The shelf life of digital humanities", by Florian Kräutli, Esther Chen and Matteo Valleriani, considers the challenge of how to preserve DH research outputs like websites, digital editions and virtual exhibitions. The problem is presented through two projects, with the second project building off the insights from the first. The first project dealt with the history of a text and its various editions, where the limitations of using bibliographic data in a relational database were discovered. Finding that $\mathrm{LD}$ and a data model based on CIDOC-CRM would better structure the hierarchies and relationships among metadata elements, these approaches were then adopted for the second project, a large-scale infrastructure initiative. Based on their experiences, the authors emphasise the need for collaboration between KO and $\mathrm{DH}$ professionals within such projects.

\section{Part III: Platforms and techniques}

The third part, Platforms and Techniques, contains three chapters that focus on specific platforms and technical interventions to support DH research.

Chapter 11, "Heritage metadata: A digital Periegesis", by Anna Foka, Kyriaki Konstantinidou, Linda Talatas, John Brady Kiesling, Elton Barker, Nasrin Mostofian, Cenk Demiroglu, Kajsa Palm, David A. McMeekin and Johan Vekselius, makes meaningful connections between the literary heritage information contained in the 1st century CE Greek travel writer Pausanias' Description of Greece and archaeological remains (sanctuaries, temples, statues, inscriptions etc.) preserved in sites and museums in present-day Greece using an open-source semantic annotation platform and LOD to enrich the location of heritage information that has been mapped using GIS. The result is a geospatially enriched digital edition of the Description of Greece that can be used to better address the traditional humanities' research questions about identity, culture, memory and social interaction, which can also be exported to other platforms directed at various audiences.

Chapter 12, "Machine learning techniques for the management of digitised collections", by Mathias Coeckelbergs and Seth van Hooland, discusses the problems and potential of topic modelling as applied to archives and how that method can help manage large collections of electronic documents. The case study applies topic modelling to a European Commission Archives sample comprising over 24,000 multilingual documents from 1958 to 1982 . It proved possible to automatically identify the topics of approximately $70 \%$ of the documents, with most failures being attributed to problems with the digitised documents, which suffer from poor optical character 
recognition (OCR). The topics were then matched against the Eurovoc thesaurus, a multilingual, multidisciplinary controlled vocabulary covering the activities of the European Union. From a DH perspective this work shows how topic modelling in the context of $\mathrm{KO}$ systems can make large datasets, initially without much metadata, meaningful for researchers using automated methods.

Chapter 13, "Exploring digital cultural heritage through browsing", by Mark M. Hall and David Walsh, addresses the shortcomings of the traditional keyword search box as a navigation interface for non-expert users visiting online culture heritage collections. The chapter surveys various solutions to facilitate user navigation of collections, including faceted search and browsing using temporal or spatial interfaces (e.g., timelines, maps) as organisational principles. In their case study, the authors built the digital museum map (DMM), an automatically generated browsing interface that allows the user to experience a virtual museum with floors and rooms that display the collection. The museum objects were organised by topic using NLP and the Getty Art and Architecture Thesaurus (AAT). While building an open-source solution, the research calls for further context-based user evaluation.

In summary, the contributed chapters provide snapshots of how information can be organised in various contexts in DH. Organising cultural heritage in digital environments is addressed in topics such as the creation and adoption of conceptual models and metadata standards (Chapters 2-6), the incorporation of LOD (Chapters 4 and 5), ways of enriching metadata (Chapters 5 and 6) and the aggregation and interoperability of metadata across cultural heritage collections (Chapters 2-6). Further highlighting the role of $\mathrm{KO}$ for $\mathrm{DH}$, these chapters discuss managing $\mathrm{DH}$ resources and DH documents for preservation and reuse (Chapters 8-10) and offer numerous examples of (semi-) automated approaches to support $\mathrm{KO}$ for improved access, discovery and navigation of materials (Chapters 5, 8, 11-13). Crucially, the chapters invite us to consider the nature of knowledge production within the humanities, and to actively work towards representing fundamental epistemic elements such as uncertainty, interpretation, context and narrative which are imported from systems and technologies adopted from outside the field (Chapter 7).

\section{Moving forward}

There are several recurring challenges in DH identified in this volume which emphasise the importance of KO for DH scholarly activities. To simplify, we might distil these challenges into two core areas where computation has long promised to surmount the limits inherent to traditional practices in the humanities: information discovery and information representation. While the two concepts are connected, since the discovery of an information object is determined by how the object is represented, we stress the perspective 
of search and access in the former and the specific challenge of how representation is constituted in the latter. The chapters in this volume, echoing the established literature, indicate that the full potential of information discovery and information representation in the field of $\mathrm{DH}$ cannot be realised without genuine transdisciplinary collaborations that actively engage with the development of DH technologies, practices and outputs. Furthermore, as part of such transdisciplinary collaborations, $\mathrm{KO}$ and its associated disciplines stand to make invaluable contributions to both information discovery and information representation in $\mathrm{DH}$.

\section{Information discovery}

Information search and retrieval are the raison d'être of KO, and $\mathrm{KO}$ systems are constantly being revisited and revised for their utility and efficiency in helping users perform these tasks. However, the usefulness of controlled vocabularies of $\mathrm{KO}$ have been a subject of debate for decades (Svenonius 1986; Rowley 1994; Gross, Taylor, and Joudrey 2015; Hjørland 2016b). This led to authorised subject index terms being neglected in the subsequent development of information retrieval systems and, as a result, today's information retrieval systems do not provide good quality subject-based access for humanities researchers (see Golub et al. 2020). Moreover, the assessment of controlled vocabularies during search has rarely been evaluated from a user perspective (see Wittek et al. 2016; Liu et al. 2017; Liu and Wacholder 2017 for exceptions). The same situation seems now to be playing itself out in the area of semantic data in cultural heritage: recent approaches in the development of semantic technologies and the application of semantic data enrichment in cultural heritage institutions are intended to expand access points to support the discovery of resource and knowledge discovery (see Wang et al., Chapter 5; Munnelly, Pandit, and Lawless 2018; Hyvönen et al. 2019; Zeng 2019). However, partly due to the disciplinary differences between the various groups designing and using such systems, as in the example of the retrieval system given above, the usefulness of semantically enriched data has not been rigorously evaluated in the context of information retrieval tasks from user perspectives.

Similarly, the search and browse interfaces of information retrieval systems affect information discovery, and their design needs to be informed by end user requirements. In addition to interfaces to IR systems such as the DMM, described in Chapter 13, also relevant here are interfaces and interactions that digitally enhance users' experience in physical museums, ranging from physical installations to mobile applications, interconnected activities and virtual/augmented/mixed (XR) reality experiences (Hornecker and Ciolfi 2019). The methodology of interaction design and participatory design from HCI studies could help reconceptualise ways of designing the tools that support DH research. Earlier efforts in this direction can be seen in the attempt by Fidel (2012) to bridge the gap between 
human information behaviour and the design of information systems. This work provides a conceptual framework and analytical tools to consider the person in a specific context or situation. Also relevant is the sub-field of interaction design within $\mathrm{HCI}$, providing a framework for the design and evaluation of interactive technologies, with emphasis on the involvement of stakeholders throughout the design life cycle (Sharp, Preece, and Rogers 2019). An example of this is co-design or participatory design visualisation framework, which deliberately involves the actors (users), activities and artefacts, using multiple methods and an iterative design approach (Dörk et al. 2020).

The adoption of $\mathrm{KO}$ for improved information discovery cannot be divorced from approaches from related disciplines that would allow for a clearer understanding of humanities scholars' information practices in specific contexts. DH scholars' information needs have to be thoroughly and continuously researched to inform all of these KO processes, models, standards and guidelines. Lessons learned from the ResearchSpace project (Chapter 7) provide a major wake-up call for user studies and participatory KO. Understanding humanities researchers is key to understanding what kinds of KO systems, processes and standards we should create and provide. Future research into $\mathrm{KO}$ in general, and into $\mathrm{KO}$ for $\mathrm{DH}$ specifically, should focus on gaining a deep understanding of the context of information needs, search, interaction and use.

\section{Information representation}

Representation is an essential component of KO. To take a simple example, a catalogue of journal articles consists of representations of each separate article. These representations may consist of descriptive information (author, title etc.) or subject information (thesauri descriptor, classification, keyword etc.). The catalogue records incorporating these representations act as surrogates for the documents themselves, abstracted and modelled into a format that is easier to process for any number of tasks, the most common of which are search and retrieval. This is not to suggest that $\mathrm{KO}$ is a purely pragmatic endeavour; the field also engages with the philosophical aspects of representation and consequences of representational devices (e.g., Olson 2002; Adler 2017). KO offers a rich collection of insights, strategies, tools and critical reflections on the representation of information and knowledge, and these stand to make valuable contributions to scholars in DH as they confront the challenges and limitations in how their objects of study are represented in the digital technologies that they employ.

Given the acknowledged connection between discovery and representation, it should be unsurprising that the ResearchSpace project (Oldman, Chapter 7) is also illustrative of the challenge of information representation for DH. ResearchSpace stands in sharp contrast to traditional databases 
that do not support historical research. Oldman demonstrates that DH research practices and the ways humanities scholars interact with their sources do not match with what is conventionally expressed in databases. To meet the diverse information needs of DH scholars, we need to reconceptualise how digital tools can be designed to support and shape their research practices, and this means including representations with greater fidelity to the epistemic paradigms under which humanists operate.

The preceding criticism has been made of LOD, given its emphasis on data at the expense of context, which Oldman directly contrasts with ResearchSpace. Nevertheless, LOD implementation is still critical to ensure interoperability and increase information access to cultural heritage data. This is why many cultural heritage institutions are moving their metadata into LOD. However, while wide adoption is crucial for LOD to be of use and add value for users, it is limited by overstretched budgets in the cultural heritage sector. Another issue identified with rapidly developing technologies, as Smith points to (Chapter 4), is that early adopters may find themselves saddled with technologies that have already been rendered obsolete by successive innovations.

Good conceptual models are an important foundation for successful LOD implementation, for making data FAIR, and for enabling interoperability of LAM metadata in general (Sugimoto, Wijesundara, Mihara, and Fukuda, Chapter 2; Vukadin and Štefanac, Chapter 3). Conceptual models such as IFLA LRM, CIDOC CRM and RiC must, on the one hand, be harmonized to ensure interoperability while, on the other hand, leave ample flexibility for new object types and different levels of descriptive granularity reflecting target collections or specific needs. The next step would be to agree on practical metadata standards and guidelines based on the harmonised conceptual models. The challenge of implementing the guidelines should be anticipated through appropriate strategies: KO professionals need to be members of DH project teams; in cases where this is not possible, there should be clear and straightforward guidelines based on models and standards from established communities in order to ensure that DH projects with little expertise in KO can nevertheless adopt and apply said guidelines. Reflections upon information representation also serve as a critical counterpoint to the representations generated by computational tools and techniques that are fundamental to further inquiry in DH. Recent developments of automated techniques, for instance, show limited usefulness for users due to the challenges in interpreting the computational models they are based on (Aletras et al. 2017; Dieng, Ruiz, and Blei 2020; Hamdi et al. 2020). More generally, computational techniques such as those used in distant reading are limited by knowledge representations hard-wired into the automated technologies.. Consider, for instance, automatic topic identification. Theoretically, automating subject determination belongs to logical positivism: a subject is considered to be a string of characters occurring above a certain threshold frequency and appearing in 
a given location, such as a title (Svenonius 2000, 46-49). But this assumes that topics, subjects or concepts have names: well-established and nonambiguous ones. Such an assumption may be well founded in, e.g., natural sciences, but much less so in the humanities and social sciences where language is often purposefully metaphorical. The positivist approach to the representation of information (where word $=$ concept) overlooks text as a complex cognitive and social phenomenon, or neglects the way cognitive understanding of text activates many knowledge sources, sustaining multiple inferences and soliciting a personal interpretation (Moens 2000, 7-10). Further research is needed to empirically test automated analytical tools, evaluating their performance for specific tasks in situated contexts (for an example of an evaluative framework for automated subject indexing, see Golub et al. 2016). It is therefore important to be critical when applying computational tools for DH research, especially in black box techniques such as topic modelling.

\section{Concluding remarks}

While KO has been finding applications in numerous areas outside its home field of information science to help address the almost universal need for organising information, we have also witnessed that, in many domains of human endeavour, information is being organised ad hoc, often resulting in systems that underperform and even effectively prevent access to data, information and knowledge. In order to help deliver the best solutions for organising information in $\mathrm{DH}$, it is important to bring the two communities of research and practice together and explore their combined potential.

The early hype about "big data", where the data at a sufficient scale simply "spoke for itself", has fortunately subsided, creating a new-found recognition that any work with data needs to take a more interdisciplinary approach, whereby different fields can share their insights on how information could be constituted and managed. As elucidated by Borgman (2015, 15), the context matters, from data creation to use, because "data, standards of evidence, forms of presentation, and research practices are deeply intertwined". Data (however it is operationalised) plays an important role in digital scholarship in the networked world: this is just as true when we consider $\mathrm{KO}$ and DH separately as when we consider their integration.

This book attempts to achieve a synergy between $\mathrm{KO}$ and $\mathrm{DH}$ by providing state-of-the-art examples of interdisciplinary projects and case studies, discussing the challenges and opportunities. The volume calls for a future in which DH research is more interdisciplinary, cutting across KO, IR, HCI, IB and other related fields and disciplines. We need to harness these complementary perspectives in order to provide the best, evidence-based KO solutions which address the complexities of DH research and, in turn, feed back into KO research. Our hope is that this volume helps set the stage for advancing $\mathrm{KO}$ in $\mathrm{DH}$ towards the mutual benefit of both. 


\section{Bibliography}

Acerbi, Alberto, Vasileios Lampos, Philip Garnett, and R. Alexander Bentley. 2013. "The Expression of Emotions in 20th Century Books." PloS One 8 (3): e59030. https://doi.org/10.1371/journal.pone.0059030

Adler, Melissa. 2017. Cruising the Library: Perversities in the Organization of Knowledge. New York, NY: Fordham University Press.

Aletras, Nikolaos, Timothy Baldwin, Jey Han Lau, and Mark Stevenson. 2017. "Evaluating Topic Representations for Exploring Document Collections." Journal of the Association for Information Science and Technology 68 (1): 154-67. https://doi.org/10.1002/asi.23574

Bates, Marcia J. 1994. "The Design of Databases and Other Information Resources for Humanities Scholars: The Getty Online Searching Project Report No. 4." Online and CD-Rom Review 18 (6): 331-40. https://doi.org/10.1108/eb024508

Bates, Marcia J. 2010. "Information.” In Encyclopedia of Library and Information Sciences, edited by Marcia J. Bates, and Mary Niles Maack, 2347-60. 3rd ed. New York, NY: CRC Press. https://pages.gseis.ucla.edu/faculty/bates/articles/ information.html

Berners-Lee, Tim, James Hendler, and Ora Lassila. 2001. "The Semantic Web." Scientific American 2001 (May): 29-37.

Blei, David M. 2012. "Topic Modeling and Digital Humanities.” Journal of Digital Humanities 2 (1): 8-11.

Borgman, Christine L. 2015. Big Data, Little Data, No Data: Scholarship in the Networked World. Cambridge MA: MIT Press.

Busa, Roberto. 1980. "The Annals of Humanities Computing: The Index Thomisticus". Computers and the Humanities 14: 83-90. https://doi.org/10.1007/ BF02403798

Case, Donald O. and Lisa M. Given, eds. 2016. Looking for Information: A Survey of Research on Information Seeking, Needs, and Behavior. Bingley: Emerald Group Publishing.

Dieng, Adji B., Francisco J. R. Ruiz, and David M. Blei. 2020. "Topic Modeling in Embedding Spaces." Transactions of the Association for Computational Linguistics 8: 439-53. https://doi.org/10.1162/tacl_a_00325

Dörk, Marian, Boris Müller, Jan-Erik Stange, Johannes Herseni, and Katja Dittrich. 2020. "Co-Designing Visualizations for Information Seeking and Knowledge Management.” Open Information Science 4 (1): 217-35. https://doi. org/10.1515/opis-2020-0102

Drucker, Johanna. 2011. "Humanities Approaches to Graphical Display." Digital Humanities Quarterly 5 (1). http://www.digitalhumanities.org/dhq/ vol/5/1/000091/000091.html

Drucker, Johanna. 2021. The Digital Humanities Coursebook. London: Routledge.

Europeana. 2021. https://www.europeana.eu/about-us

Fidel, Raya. 2012. Human Information Interaction: An Ecological Approach to Information Behavior. Cambridge MA: MIT Press.

Flanders, Julia, and Fotis Jannidis, eds. 2018. The Shape of Data in Digital Humanities: Modeling Texts and Text-based Resources. London: Routledge.

Gardiner, Eileen, and Roland G. Musto. 2015. The Digital Humanities: A Primer for Students and Scholars. Cambridge: Cambridge University Press.

Gitelman, Lisa. 2013. 'Raw Data' Is an Oxymoron. Cambridge MA: MIT Press. 
Glushko, Robert J. 2016. The Discipline of Organizing: Professional Edition. 4th ed. O'Reilly Media: Sebastopol, CA.

Golub, Koraljka. 2019. "Automatic Subject Indexing of Text." Knowledge Organization 46 (2): 104-121. https://doi.org/10.5771/0943-7444-2019-2-104. Also available in ISKO Encyclopedia of Knowledge Organization, edited by Birger Hjørland and Claudio Gnoli. https://www.isko.org/cyclo/automatic

Golub, Koraljka. 2020. "Automatic Identification of Topics: Applications and Challenges." In Doing Digital Humanities: Concepts, Approaches, Cases, edited by Joacim Hansson and Jonas Svensson, 5-26. Växjö: Linnaeus University Press.

Golub, Koraljka, Dagobert Soergel, George Buchanan, Douglas Tudhope, Marianne Lykke, and Debra Hiom. 2016. "A Framework for Evaluating Automatic Indexing or Classification in the Context of Retrieval." Journal of the Association for Information Science and Technology 67 (1), 3-16. https://doi. org/10.1002/asi.23600

Golub, Koraljka, Henk Muller, and Emma Tonkin, E. 2014. "Technologies for Metadata Extraction." In Handbook of Metadata, Semantics and Ontologies, edited by Miguel-Angel Sicilia, 487-522. Hackensack, NJ: World Scientific.

Golub, Koraljka, Jukka Tyrkkö, Joachim Hansson, and Ida Ahlström. 2020. "Subject Indexing in Humanities : A Comparison between a Local University Repository and an International Bibliographic Service". Journal of Documentation 76 (6): 1193-1214. https://doi.org/10.1108/JD-12-2019-0231

Gross, Tina, Arlene G. Taylor, and Daniel N. Joudrey. 2015. "Still a Lot to Lose: The Role of Controlled Vocabulary in Keyword Searching." Cataloging \& Classification Quarterly 53 (1): 1-39. https://doi.org/10.1080/01639374.2014.917447

Hamdi, Ahmed, Axel Jean-Caurant, Nicolas Sidère, Mickaël Coustaty, and Antoine Doucet. 2020. "Assessing and Minimizing the Impact of OCR Quality on Named Entity Recognition." In Digital Libraries for Open Knowledge. TPDL 2020. Lecture Notes in Computer Science, vol. 12246, edited by Mark Hall, Tanja Merčun, Thomas Risse, and Fabien Duchateau, 87-101. Cham: Springer. https:// doi.org/10.1007/978-3-030-54956-5_7

Haeselin, David. 2017. "Concordance”. In ArchBook. Last modified April 1, 2019. http://drc.usask.ca/projects/archbook/concordance.php

Hawkins, Kevin, Michelle Dalmau, Elli Mylonas, and Syd Bauman. 2018. Best Practices for TEI in Libraries. https://tei-c.org/extra/teiinlibraries/4.0.0/ bptl-driver.html

Hider, Philip, Barney Dalgarno, Sue Bennett, Ying-Hsang Liu, Carole Gerts, Carla Daws, Barbara Spiller, Pru Mitchell, Robert Parkes, and Raylee Macaulay. 2016. "Reindexing a Research Repository from the Ground up: Adding and Evaluating Quality Metadata.” Australian Academic and Research Libraries 47 (2): 61-75. https://doi.org/10.1080/00048623.2016.1204589

Hjørland, Birger. 2012. "Knowledge Organization = Information Organization?." Advances in Knowledge Organization 13: 8-14.

Hjørland, Birger. 2016a. "Knowledge organization (KO)." Knowledge Organization 43 (6): 475-85. Also available in ISKO Encyclopedia of Knowledge Organization, edited by Birger Hjørland and Claudio Gnoli. https://www.isko.org/cyclo/ knowledge_organization.

Hjørland, Birger. 2016b. "Does the Traditional Thesaurus Have a Place in Modern Information Retrieval?." Knowledge Organization 43 (3): 145-59. https://doi. org/10.5771/0943-7444-2016-3-145 
Hjørland, Birger. 2017. "Subject (of Documents)." Knowledge Organization 44 (1): 55-64. https://doi.org/10.5771/0943-7444-2017-1-55

Hornecker, Eva, and Luigina Ciolfi. 2019. "Human-Computer Interactions in Museums." Synthesis Lectures on Human-Centered Informatics 12 (2): i-153. https://doi.org/10.2200/S00901ED1V01Y201902HCI042

Hyvönen, Eero, Petri Leskinen, Minna Tamper, Heikki Rantala, Esko Ikkala, Jouni Tuominen, and Kirsi Keravuori. 2019. "BiographySampo - Publishing and Enriching Biographies on the Semantic Web for Digital Humanities Research." In The Semantic Web. ESWC 2019. Lecture Notes in Computer Science, vol. 11503, edited by Pascal Hitzler, Miriam Fernández, Krzysztof Janowicz, Amrapali Zaveri, Alasdair J.G. Gray, Vanessa Lopez, Armin Haller, and Karl Hammar. Cham: Springer. https://doi.org/10.1007/978-3-030-21348-0_37

International Council on Archives. 2019. Records in Contexts. A Conceptual Model for Archival Description. Consultation Draft v0.2 (preview). December 2019. https://www.ica.org/sites/default/files/ric-cm-0.2_preview.pdf

International Federation of Library Associations. 2017. IFLA Library Reference Model, A Conceptual Model for Bibliographic Information. https://www.ifla.org/ files/assets/cataloguing/frbr-lrm/ifla-lrm-august-2017_rev201712.pdf

International Standards Organization. 2006. CIDOC Conceptual Reference Model. http://www.cidoc-crm.org/

Kansa, Eric, and Sarah Whitcher Kansa. 2021. "Digital Data and Data Literacy in Archaeology. Now and in the New Decade." Advances in Archaeological Practice 9 (1): 81-85. https://doi.org/10.1017/aap.2020.5

Kilgour, Frederick G. 1939. "A new Punched Card for Circulation Records.” Library Journal 64 (4): 131-133.

Kirschenbaum, Matthew G. 2010. "What is Digital Humanities and What's it Doing in English Departments?". ADE Bulletin 150: 55-61.

Liu, Ying-Hsang, Paul Thomas, Marijana Bacic, Tom Gedeon, and Xindi Li. 2017. "Natural Search User Interfaces for Complex Biomedical Search: An Eye Tracking Study." Journal of the Australian Library and Information Association 66 (4): 364-81. https://doi.org/10.1080/24750158.2017.1357915

Liu, Ying-Hsang, and Nina Wacholder. 2017. "Evaluating the Impact of MeSH (Medical Subject Headings) Terms on Different Types of Searchers." Information Processing and Management 53 (4): 851-70. https://doi.org/10.1016/j.ipm.2017.03.004

Luhn, Hans Peter. 1960. "Keyword-In-Context Index for Technical Literature". American Documentation 11 (4): 288-95.

Mayernik, Matthew S. 2020. "Metadata”. Knowledge Organization 47 (8): 696-713. Also available in ISKO Encyclopedia of Knowledge Organization, edited by Birger Hjørland and Claudio Gnoli. https://www.isko.org/cyclo/metadata

Moens, Marie-Francine. 2000. Automatic indexing and abstracting of document texts. Boston: Kluwer.

Moretti, Franco. 2000. "Conjectures on World Literature”. New Left Review 238 (1). https://newleftreview.org/issues/iil/articles/franco-moretti-conjectures-onworld-literature

Munnelly, Gary, Harshvardhan J. Pandit, and Séamus Lawless. 2018. "Exploring Linked Data for the Automatic Enrichment of Historical Archives." In The Semantic Web: ESWC 2018 Satellite Events. ESWC 2018. Lecture Notes in Computer Science, vol. 11155, edited by Aldo Gangemi, 423-33. Cham: Springer. https://doi.org/10.1007/978-3-319-98192-5_57 
Münster, Sander. 2019. "Digital Heritage as a Scholarly Field-Topics, Researchers, and Perspectives from a Bibliometric Point of View." Journal on Computing and Cultural Heritage 12 (3): 22.1-22.27. https://doi.org/10.1145/3310012

Nyhan, Julianne, and Andrew Flinn. 2016. Computation and the Humanities: Towards an Oral History of Digital Humanities. Cham: Springer.

Olson, Hope A. 2002. The Power to Name: Locating the Limits of Subject Representation in Libraries. Dordrecht: Kluwer.

Oxford online dictionary, s.v. "Digital Humanities" accessed February 10, 2020, https://www.lexico.com/definition/digital_humanities

Resource Description and Access (RDA). 2021. https://www.loc.gov/aba/rda/

Rowley, Jennifer. 1994. "The Controlled versus Natural Indexing Languages Debate Revisited: A Perspective on Information Retrieval Practice and Research.” Journal of Information Science 20 (2): 108-18. https://doi.org/10.1177/016555159402000204

Saracevic, Tefko. 2009. "Information science." In Encyclopedia of Library and Information Science, edited by Marcia J. Bates and Mary Niles Maack, 25702586. 3rd ed. New York, NY: CRC Press. https://tefkos.comminfo.rutgers.edu/ SaracevicInformationScienceELIS2009.pdf

Schreibman, Susan, Ray Siemens, and John Unsworth. 2016. A New Companion to Digital Humanities. 2nd ed. Hoboken, NJ: John Wiley \& Sons.

Sharp, Helen, Jennifer Preece, and Yvonne Rogers. 2019. Interaction Design: Beyond Human-Computer Interaction. 5th ed. Hoboken, NJ: John Wiley \& Sons.

Svenonius, Elaine. 1986. "Unanswered Questions in the Design of Controlled Vocabularies." Journal of the American Society for Information Science 37 (5): 331-40. https://doi.org/10.1002/(SICI)1097-4571(198609)37:5<331::AID-ASI8>3.0. CO;2-E

Svenonius, Elaine. 2000. The Intellectual Foundation of Information Organization. Cambridge, MA: MIT Press.

Sula, Chris Alen, and Heather V. Hill. 2019. "The Early History of Digital Humanities: An Analysis of Computers and the Humanities (1966-2004) and Literary and Linguistic Computing (1986-2004)." Digital Scholarship in the Humanities 34 (1): 190-206. https://doi.org/10.1093/1lc/fqz072

Terras, Melissa, Julianne Nyhan, and Edward Vanhoutte, eds. 2013. Defining Digital Humanities. A Reader. London: Routledge.

Text Encoding Initiative. 2017. Text Encoding Initiative. https://tei-c.org/

Vanhoutte, Edward. 2013. "The Gates of Hell: History and Definition of Digital Humanities Computing." In Defining Digital Humanities. A Reader, edited by Melissa Terras, Julianne Nyhan, and Edward Vanhoutte. 119-56. London: Routledge.

Wittek, Peter, Ying-Hsang Liu, Sándor Darányi, Tom Gedeon, and Ik Soo Lim. 2016. "Risk and Ambiguity in Information Seeking: Eye Gaze Patterns Reveal Contextual Behavior in Dealing with Uncertainty." Human-Computer Interaction. Frontiers in Psychology 7:1790. https://doi.org/10.3389/fpsyg.2016.01790

W3C Incubator Group. 2011. Library Linked Data Incubator Group: Datasets, Value Vocabularies, and Metadata Element Sets, W3C Incubator Group Report 25 October 201. https://www.w3.org/2005/Incubator/1ld/XGR-1ld-vocabdataset-20111025/\# Introduction:_Scope_and_Definitions

Wilkinson, Mark D. et al. 2016. "The FAIR Guiding Principles for Scientific Data Management and Stewardship." Scientific Data 3: 160018. https://doi.org/10.1038/ sdata. 2016.18 
22 Koraljka Golub et al.

World Wide Web Consortium. 2015. Linked Data. https://www.w3.org/standards/ semanticweb/data.html

Zeng, Marcia Lei. 2019. "Semantic Enrichment for Enhancing LAM Data and Supporting Digital Humanities. Review Article." El Profesional de La Información 28 (1): 1-35. https://doi.org/10.3145/epi.2019.ene.03

Zeng, Marcia Lei, and Jian Qin. 2016. Metadata. 2nd ed. Chicago, IL: ALA Neal-Schuman. 